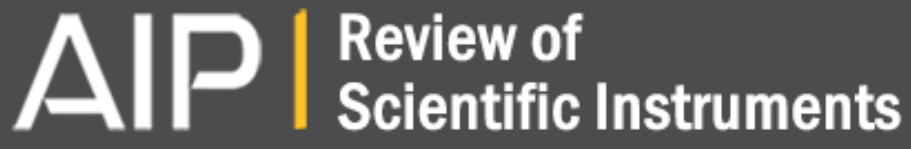

\section{Sub-nanosecond time-resolved ambient-pressure X-ray photoelectron spectroscopy setup for pulsed and constant wave X-ray light sources}

Andrey Shavorskiy, Stefan Neppl, Daniel S. Slaughter, James P. Cryan, Katrin R. Siefermann, Fabian Weise, Ming-Fu Lin, Camila Bacellar, Michael P. Ziemkiewicz, loannis Zegkinoglou, Matthew W. Fraund, Champak Khurmi, Marcus P. Hertlein, Travis W. Wright, Nils Huse, Robert W. Schoenlein, Tolek Tyliszczak, Giacomo Coslovich, Joseph Robinson, Robert A. Kaindl, Bruce S. Rude, Andreas Ölsner, Sven Mähl, Hendrik Bluhm, and Oliver Gessner

Citation: Review of Scientific Instruments 85, 093102 (2014); doi: 10.1063/1.4894208

View online: http://dx.doi.org/10.1063/1.4894208

View Table of Contents: http://scitation.aip.org/content/aip/journal/rsi/85/9?ver=pdfcov

Published by the AIP Publishing

Articles you may be interested in

A setup for resonant inelastic soft $x$-ray scattering on liquids at free electron laser light sources

Rev. Sci. Instrum. 83, 123109 (2012); 10.1063/1.4772685

Time-resolved soft $\mathrm{x}$-ray spectra from laser-produced Cu plasmaa)

Rev. Sci. Instrum. 83, 10E138 (2012); 10.1063/1.4739071

Photosynthetic Dioxygen Formation Monitored by Time-Resolved X-Ray Spectroscopy

AIP Conf. Proc. 882, 301 (2007); 10.1063/1.2644507

Novel time-of-flight electron spectrometer optimized for time-resolved soft-x-ray photoelectron spectroscopy Rev. Sci. Instrum. 77, 043105 (2006); 10.1063/1.2194475

A setup for ultrafast time-resolved x-ray absorption spectroscopy

Rev. Sci. Instrum. 75, 24 (2004); 10.1063/1.1633003

You don't

still use this

cell phone

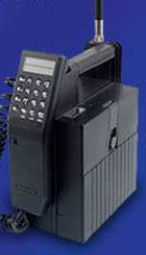

or this computer

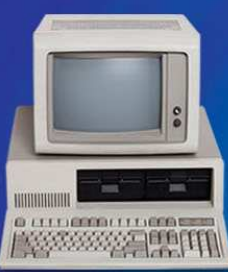

Why are you

still using an

AFM designed in the 80 's?
It is time to upgrade your AFM Minimum $\$ 20,000$ trade-in discount for purchases before August 31st

Asylum Research is today's technology leader in AFM 


\title{
Sub-nanosecond time-resolved ambient-pressure X-ray photoelectron spectroscopy setup for pulsed and constant wave $X$-ray light sources
}

\author{
Andrey Shavorskiy, ${ }^{1}$ Stefan Neppl, ${ }^{2}$ Daniel S. Slaughter, ${ }^{1}$ James P. Cryan, ${ }^{2}$ \\ Katrin R. Siefermann, ${ }^{2, a)}$ Fabian Weise, ${ }^{2, b)}$ Ming-Fu Lin, ${ }^{2, c)}$ Camila Bacellar, ${ }^{2}$ \\ Michael P. Ziemkiewicz, ${ }^{2}$ loannis Zegkinoglou, ${ }^{1}$ Matthew W. Fraund, ${ }^{2}$ Champak Khurmi, ${ }^{2, d)}$ \\ Marcus P. Hertlein, ${ }^{3, e)}$ Travis W. Wright, ${ }^{2}$ Nils Huse, ${ }^{2,4}$ Robert W. Schoenlein, ${ }^{2}$ \\ Tolek Tyliszczak, ${ }^{3}$ Giacomo Coslovich, ${ }^{5}$ Joseph Robinson, ${ }^{5,6}$ Robert A. Kaindl, ${ }^{5}$ \\ Bruce S. Rude, ${ }^{1}$ Andreas Ölsner, ${ }^{7}$ Sven Mähl, ${ }^{8}$ Hendrik Bluhm, ${ }^{1}$ and Oliver Gessner ${ }^{2, f)}$ \\ ${ }^{1}$ Chemical Sciences Division, Lawrence Berkeley National Laboratory, Berkeley, California 94720, USA \\ ${ }^{2}$ Ultrafast X-ray Science Laboratory, Chemical Sciences Division, Lawrence Berkeley National Laboratory, \\ Berkeley, California 94720, USA \\ ${ }^{3}$ Advanced Light Source, Lawrence Berkeley National Laboratory, Berkeley, California 94720, USA \\ ${ }^{4}$ Physics Department, University of Hamburg and Max-Planck Institute for Structure and Dynamics of Matter, \\ 22761 Hamburg, Germany \\ ${ }^{5}$ Materials Sciences Division, Lawrence Berkeley National Laboratory, Berkeley, California 94720, USA \\ ${ }^{6}$ SLAC National Accelerator Laboratory, Menlo Park, California 94025, USA \\ ${ }^{7}$ Surface Concept GmbH, 55124 Mainz, Germany \\ ${ }^{8}$ SPECS Surface Nano Analysis GmbH, 13355 Berlin, Germany
}

(Received 31 July 2014; accepted 18 August 2014; published online 2 September 2014)

\begin{abstract}
An apparatus for sub-nanosecond time-resolved ambient-pressure X-ray photoelectron spectroscopy studies with pulsed and constant wave X-ray light sources is presented. A differentially pumped hemispherical electron analyzer is equipped with a delay-line detector that simultaneously records the position and arrival time of every single electron at the exit aperture of the hemisphere with $\sim 0.1 \mathrm{~mm}$ spatial resolution and $\sim 150 \mathrm{ps}$ temporal accuracy. The kinetic energies of the photoelectrons are encoded in the hit positions along the dispersive axis of the two-dimensional detector. Pumpprobe time-delays are provided by the electron arrival times relative to the pump pulse timing. An average time-resolution of $(780 \pm 20)$ ps $(F W H M)$ is demonstrated for a hemisphere pass energy $E_{p}$ $=150 \mathrm{eV}$ and an electron kinetic energy range $K E=503-508 \mathrm{eV}$. The time-resolution of the setup is limited by the electron time-of-flight (TOF) spread related to the electron trajectory distribution within the analyzer hemisphere and within the electrostatic lens system that images the interaction volume onto the hemisphere entrance slit. The TOF spread for electrons with $K E=430 \mathrm{eV}$ varies between $\sim 9 \mathrm{~ns}$ at a pass energy of $50 \mathrm{eV}$ and $\sim 1 \mathrm{~ns}$ at pass energies between $200 \mathrm{eV}$ and $400 \mathrm{eV}$. The correlation between the retarding ratio and the TOF spread is evaluated by means of both analytical descriptions of the electron trajectories within the analyzer hemisphere and computer simulations of the entire trajectories including the electrostatic lens system. In agreement with previous studies, we find that the by far dominant contribution to the TOF spread is acquired within the hemisphere. However, both experiment and computer simulations show that the lens system indirectly affects the time resolution of the setup to a significant extent by inducing a strong dependence of the angular spread of electron trajectories entering the hemisphere on the retarding ratio. The scaling of the angular spread with the retarding ratio can be well approximated by applying Liouville's theorem of constant emittance to the electron trajectories inside the lens system. The performance of the setup is demonstrated by characterizing the laser fluence-dependent transient surface photovoltage response of a laser-excited Si(100) sample. () 2014 AIP Publishing LLC. [http://dx.doi.org/10.1063/1.4894208]
\end{abstract}

\section{INTRODUCTION}

Time-domain X-ray spectroscopy techniques are gaining increasing attention in a variety of scientific commu-

\footnotetext{
a) Present address: Leibniz Institute of Surface Modification, 04138 Leipzig, Germany.

b) Present address: Berliner Glas KGaA, 12347 Berlin, Germany.

c) Present address: Department of Chemistry, University of Illinois at UrbanaChampaign, Urbana, Illinois 61801, USA.

d) Present address: Centre for Quantum Dynamics, Griffith University, Nathan, Australia.

e) Present address: Coherent Inc., Santa Clara, California 95054, USA.

f) Author to whom correspondence should be addressed. Electronic mail: ogessner@lbl.gov.
}

nities due to their potential to reveal details of fast dynamic processes in matter that are not accessible in static $\mathrm{X}$-ray experiments and which cannot be addressed with traditional time-resolved all-optical methods. The prospect to probe changes in local electronic structures and chemical transformations in real time with element specificity is a major driver for the development of laboratory-based ${ }^{1-4}$ and accelerator-based ${ }^{5-8}$ ultrafast $\mathrm{X}$-ray techniques that complement both energy-domain X-ray spectroscopy efforts at synchrotrons as well as time-domain spectroscopy techniques using ultraviolet, visible, and infrared lasers. In the regime of picosecond to nanosecond time-resolved X-ray 
measurements, synchrotron light sources are particularly attractive due to their inherent timing structure with X-ray pulse durations on the order of $\sim 30-100 \mathrm{ps},{ }^{9-13}$ pulse repetition rates at $\mathrm{MHz}$ levels, continuous and wide range photon energy tunability, excellent long-term stability, and the ready availability of a large variety of mature experimental X-ray techniques.

Generally, time-resolved synchrotron experiments can be divided into two classes: time-discriminating and timerecording ("tagging"). In the time-discriminating approach, an external stimulus, such as a laser is synchronized to a specific X-ray pulse with a fixed time-delay and the detector is gated to suppress signals from most or all other synchrotron bunches. ${ }^{10-16}$ This approach gives access to very high time resolution reaching the femtosecond regime ${ }^{8,11,17}$ but uses only a small fraction of the available X-ray flux. In the timerecording approach - which is employed in this study - the external stimulus may or may not be synchronized to the synchrotron pulses and the detector is not gated. Instead, the arrival times ("time stamps") of the detector signals relative to the external stimulus are recorded and the data are sorted according to their time stamps. ${ }^{9,18-21}$ This approach can, in principle, make use of the entire X-ray flux (depending on the time delay range of interest) with an intrinsic time resolution that is limited by the detector ${ }^{18,19}$ and/or the spectrometer characteristics..$^{9,22}$

Here, a sub-ns time-resolved ambient pressure XPS setup is presented that combines a fast two-dimensional delay-line detector with $\sim 150$ ps time resolution with a hemispherical electron analyzer that is equipped with a differentially pumped electrostatic lens system. ${ }^{23}$ The time-resolved (tr)XPS setup can be operated with pulsed and constant wave (CW) X-ray light sources. When synchronized to the electron bunch train of the Advanced Light Source (ALS) storage ring, the apparatus can readily distinguish $\mathrm{X}$-ray signals from different bunches within the $\sim 500 \mathrm{MHz}$ ALS multi-bunch pattern. This capability opens the route to perform bunch length-limited picosecond time-resolved ambient pressure Xray photoelectron spectroscopy experiments at synchrotron radiation light sources with high efficiency, during all storage ring operating modes, and with modest requirements on existing beamline facilities.

The temporal resolution of the system is characterized and theoretically analyzed for a wide range of retarding ratios. The electron trajectories within the ambient pressure compatible electrostatic lens system induce a pronounced retarding ratio dependence of the system's temporal resolution, which can be well approximated by Liouville's theorem of constant emittance. ${ }^{24}$ The tr-XPS setup is used to characterize the fluence-dependence of the laser-induced transient surface photovoltage (SPV) effect in a $\mathrm{Si}(100)$ sample, clearly demonstrating a monotonic increase of the SPV decay time scale when the laser fluence is increased from $\sim 1 \mathrm{~nJ} / \mathrm{cm}^{2}$ to $\sim 160 \mu \mathrm{J} / \mathrm{cm}^{2}$.

\section{DESIGN}

Fig. 1 shows a schematic of the laser-pump/X-ray-probe photoelectron spectroscopy setup. Experiments are performed using the ambient pressure photoelectron spectroscopy endstation (APPES-2) at the molecular environmental sciences beamline 11.0.2 of the Advanced Light Source. ${ }^{25}$ The central element of the setup is a hemispherical electron energy analyzer (SPECS Phoibos 150 NAP) equipped with a fast two-dimensional delay-line detector ${ }^{26}$ (DLD, Surface Concept, SC DLD-4242-H9). The analyzer is equipped with a system of differentially pumped electrostatic lenses, enabling photoemission measurements at ambient pressures up to several Torr. ${ }^{23,27,28}$ The DLD detector records both the hit position and arrival time of every single electron at the exit of the hemisphere. The photoelectron kinetic energies are encoded in the hit position on the detector in the dispersive plane of the analyzer. The electron arrival time is measured with a precision of $\sim 150 \mathrm{ps}^{29}$ relative to a fixed external trigger signal (Fig. 1, blue solid line) provided by a gate and delay generator (Stanford Research Systems, DG 535). The same signal generator provides a trigger signal for the laser (Coherent Evolution 70) that is used for the laser-pump/Xray-probe experiment presented below (Fig. 1, green solid line).

The key concept of the setup is that the simultaneous recording of the kinetic energy and the time stamp of every electron relative to the trigger signal of the laser constitutes a time-resolved XPS measurement in which the pumpprobe delay is not controlled but recorded ("tagged"). Timeresolved XPS spectra are recovered by using a time-to-digital converter (TDC) to sort the kinetic energies of all electrons into channels corresponding to different pump-probe time delays (Fig. 1, bottom). Similar concepts were previously applied in the works of Gießel et al. ${ }^{9}$ and Bergeard et al. ${ }^{18} \mathrm{~A}$ distinct feature of the system described here is the use of a fast, high-resolution two-dimensional detector. The simultaneous recording of 1024 energy channels along the dispersive axis enables the acquisition of high-resolution tr-XPS spectra across a large (typically tens of eV) kinetic energy window without the need to sweep the analyzer and the high time resolution enables measurements at the TOF spread limit of the XPS analyzer. The total data acquisition times of the (sub-)nanosecond tr-XPS measurements presented here range from $\sim 5$ min (Fig. 3) to $\sim 100$ min (Figs. 5 and 6) including all spectra at all time delays. Additionally, to the best of our knowledge, this is the first performance characterization of an ambient pressure XPS setup in a (sub-)nanosecond tr-XPS experiment.

\section{COARSE ABSOLUTE TIMING CALIBRATION}

The absolute time correlation between the recorded XPS spectra and the impact of the pump laser pulse on the sample, i.e., the position of "time zero" in the time-resolved XPS spectra, is generally unknown. It can be determined either by using a sample with a known XPS response to the pump-laser pulse or, more generally, by imposing a well-defined transient change on the XPS spectrum with a known time structure. Here, a rectangular low voltage pulse (amplitude $1.5 \mathrm{~V}$, width $500 \mathrm{~ns}$, rise time $<5 \mathrm{~ns}$ ) is applied to the sample for a coarse time zero calibration (Fig. 1, red solid line). Fig. 2 shows the 


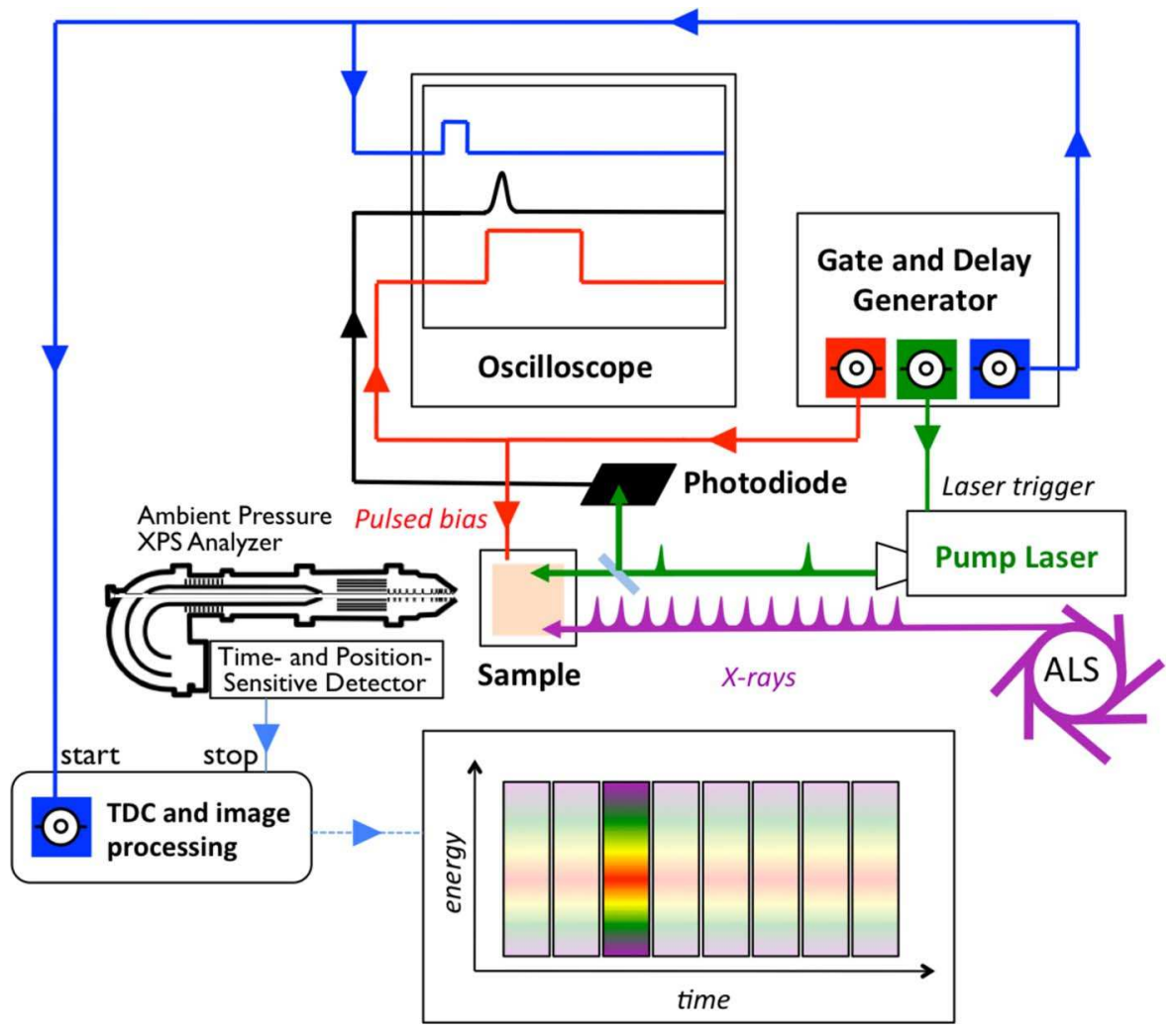

FIG. 1. Timing scheme of the tr-XPS apparatus. See text for details.

resulting tr-XPS trace from a gold foil in the region of the $\mathrm{Au}$ $4 f_{7 / 2}$ photoelectron line. The $2 \mathrm{D}$ map in Fig. 2(a) is a falsecolor representation of the tr-XPS data with the kinetic energy decreasing along the vertical axis and time progressing along the horizontal axis. The detector was not synchronized to the ALS bunch structure in this measurement; the pulsed synchrotron radiation effectively acts as a CW X-ray source, leading to a continuous XPS intensity along the time axis. Note that the plot shows only a short range of time delays close to the voltage pulse. The total range of recorded delays (a)
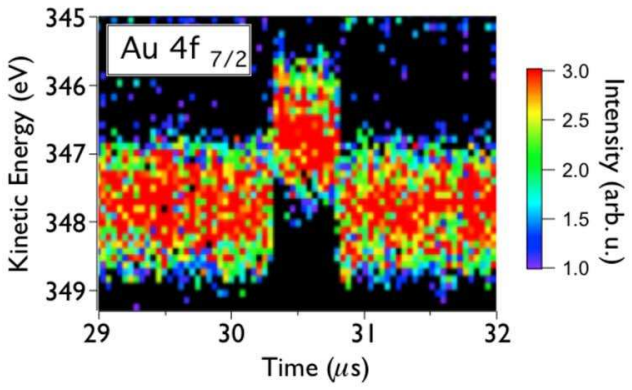

(c)

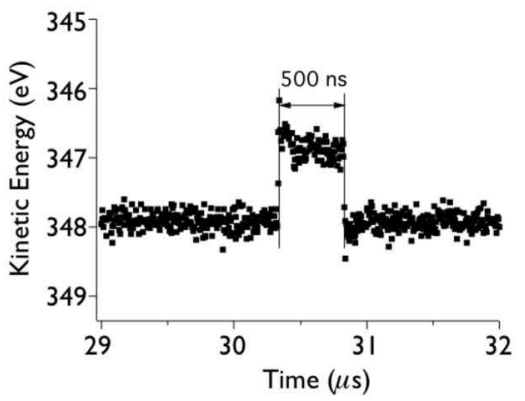

(b)

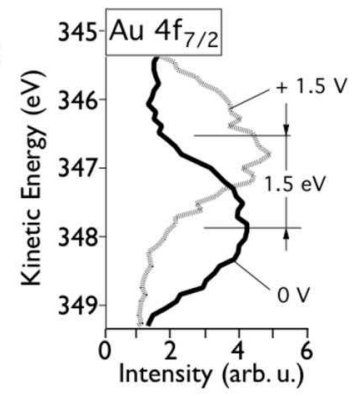

FIG. 2. (a) False-color representation of tr-XPS timing calibration spectra using the $\mathrm{Au} 4 f_{7 / 2}$ line from a gold foil. Note the transient shift by $1.5 \mathrm{eV}$ due to the pulsed sample bias voltage. (b) Examples of individual XPS-spectra measured at time stamps of $29.5 \mu \mathrm{s}$ (black) and $30.5 \mu \mathrm{s}$ (gray). (c) Time-dependent position of $\mathrm{Au} 4 f_{7 / 2}$ peak obtained from the data in (a). 
is limited by the number of TDC time channels and the time step per channel. The time-dependent sample bias voltage appears as a transient shift of the XPS spectrum to $1.5 \mathrm{eV}$ lower kinetic energies within a time window of $500 \mathrm{~ns}$ centered near the $\sim 30.5 \mu$ s time stamp. This effect is illustrated in more detail in Figs. 2(b) and 2(c). Fig. 2(b) compares Au $4 f_{7 / 2}$ peaks recorded at time stamps of $29.5 \mu \mathrm{s}$ (black) and $30.5 \mu \mathrm{s}$ (gray), which are shifted by $1.5 \mathrm{eV}$ with respect to each other. Peak positions obtained by fitting each spectrum in Fig. 2(a) to a pseudo-Voigt line shape are plotted versus time in Fig. 2(c). The sharp shift of the gold peaks to lower kinetic energies is clearly observed at $30.331 \pm 0.005 \mu \mathrm{s}$. The relative timing between the pump laser and the voltage bias pulse is derived by measuring the delay between the voltage pulse and a photodiode signal on a fast oscilloscope (Fig. 1, red and black solid lines, respectively). In this way, time zero in the tr-XPS experiments can be defined with a precision of $\sim 5 \mathrm{~ns}$ by taking all optical and electronic signal path length differences into account.

\section{TEMPORAL RESOLUTION}

The temporal resolution of the setup for pump-probe experiments is defined by three main contributions: (i) the pulse width of the pump-laser, (ii) the time resolution of the detector-TDC combination $\left(150 \mathrm{ps}^{29}\right)$, and (iii) the TOF spread of electrons within the electron analyzer. ${ }^{9,22}$ When using a picosecond laser system, the by far dominating factor is the finite electron TOF distribution (iii), which usually extends across nanosecond time ranges.

XPS signals arising from single $\sim 70$ ps long X-ray pulses of the $\mathrm{ALS}^{13}$ were used to determine the electron TOF-induced contribution to the overall temporal resolution of the setup. Fig. 3(a) shows a series of tr-XPS spectra of $\mathrm{N} 3 / \mathrm{Ru} 535 \mathrm{Ru}$-dye molecules adsorbed on a film of $\mathrm{ZnO}$ nanocrystals ${ }^{30,31}$ supported by a $\mathrm{Si}(100)$ wafer. All spectra at all time delays were recorded simultaneously at a photon energy of $640 \mathrm{eV}$. The detected kinetic energy range was chosen in a featureless region of the XPS spectrum around $K E$ $=500 \mathrm{eV}$ as indicated in Fig. 3(b). The horizontal axis of the 2D grayscale map corresponds to the electron kinetic energy and the vertical axis to the electron detection time. For these calibration measurements, the trigger for the detector system was synchronized to the ALS bunch structure. The bright tilted stripes in Fig. 3(a) are XPS spectra generated by different ALS bunches. The tilt originates from the different travel times of electrons with different kinetic energies through the XPS spectrometer, leading to different arrival times at the detector. The time difference between most spectra corresponds to the $2 \mathrm{~ns}$ bunch-to-bunch delay of the ALS multibunch operating mode. The well-isolated and most intense spectrum is generated by the so-called camshaft bunch. ${ }^{32}$

Fig. 3(c) shows the projection of a single $150 \mathrm{meV}$ wide slice (blue vertical line in Fig. 3(a)) onto the time delay axis. The overall time resolution of the system is derived by fitting the time-structures of a series of these slices covering the $K E$ range $503-508 \mathrm{eV}$ to Gaussian profiles as indicated in Fig. 3(c). The average temporal width of the camshaft sig-

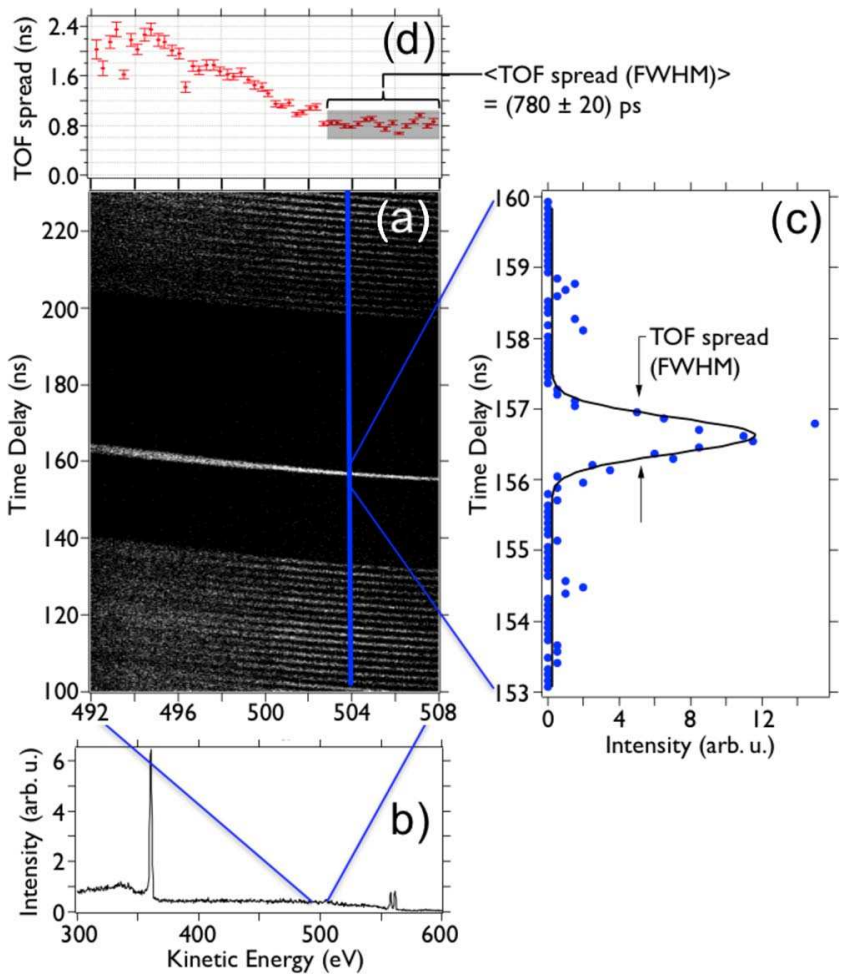

FIG. 3. Determination of the temporal resolution. (a) Time-resolved XPS spectra of N3 dye molecules adsorbed to $\mathrm{ZnO}$ nanocrystals. The bright stripes are grayscale representations of tr-XPS spectra emerging from single ALS bunches. (b) Photoemission spectrum indicating the spectral range covered by (a). (c) The TOF spread of electrons with the same kinetic energy traversing the analyzer on different trajectories is determined by fitting the temporal profile of the camshaft tr-XPS spectrum for a narrow $(150 \mathrm{meV})$ energy range (blue line in (a)) to a Gauss curve (solid black line in (c)). (d) Kinetic energy dependence of the TOF spread, averaged over two neighboring values. The average TOF spread in the $503-508 \mathrm{eV}$ kinetic energy range is $(780 \pm 20)$ ps. The photon energy is $640 \mathrm{eV}$.

nal across this $5 \mathrm{eV}$ wide energy window is $(780 \pm 20) \mathrm{ps}$ (Fig. 3(d)). To the best of our knowledge, this is the first time that a sub-ns time resolution has been demonstrated in a tr-XPS setup using the time-tagging technique. Performing the same fit procedure for slices at kinetic energies $K E$ $=492-508 \mathrm{eV}$ leads to a distribution of $K E$-dependent TOF spreads shown in Fig. 3(d) (each data point is averaged over two neighboring values). Note that the TOF spread varies by a factor of 2-3 across the $K E$ window. This variation is due to the fact that electrons with different kinetic energies traverse the spectrometer on different trajectories that depend on the electrostatic lens settings, the retarding voltage, and the hemisphere pass energy, which will be discussed in more detail below.

Fig. 4(a) shows the measured TOF spread (red squares) for XPS spectra recorded with a GaAs sample at a photon energy of $490 \mathrm{eV}$, a nominal kinetic energy of $K E=430 \mathrm{eV}$, and hemisphere pass energies $E_{p}$ varying between $50 \mathrm{eV}$ and $400 \mathrm{eV}$ (spanning retarding ratios $K E / E_{p}$ between 8.6 and 1.1). Note the double logarithmic representation. The spreads were derived by averaging measured TOF spreads over $2 \mathrm{eV}$ wide kinetic energy regions to ensure that the results are not affected by single outliers and that they are relevant with respect to applications, which usually require the use of 


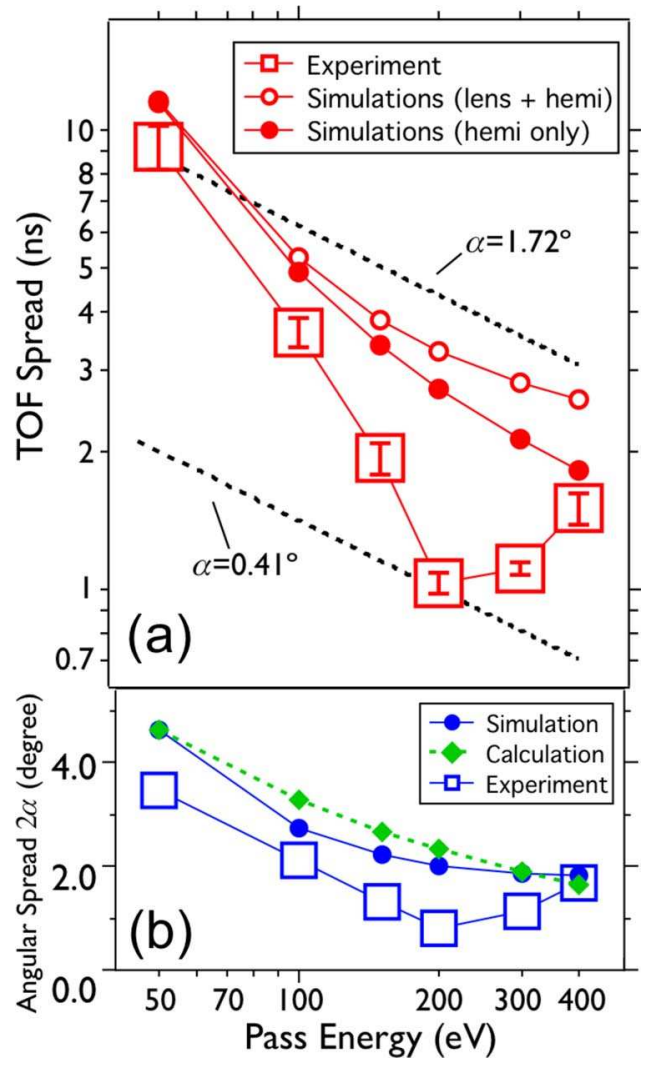

FIG. 4. Pass energy dependence of (a) the temporal resolution (FWHM) and (b) the hemisphere entrance angle spread (FWHM) of the tr-XPS setup. Open squares indicate experimental values, open and solid circles the results of a computer simulation, and solid diamonds estimates based on the theorem of constant emittance along the electron trajectories. Solid and open circles in (a) correspond to TOF spreads within the hemisphere only and across the entire electron flight paths, respectively. The nominal electron kinetic energy is $430 \mathrm{eV}(\mathrm{h} v=490 \mathrm{eV})$

extended regions of the XPS spectrum. The TOF spread drops rapidly with increasing pass energy. For $E_{p} \geq 200 \mathrm{eV}$ it is nearly constant at $\sim 1-2 \mathrm{~ns}$ (FWHM) and almost an order of magnitude smaller than for $E_{p}=50 \mathrm{eV}(\sim 9 \mathrm{~ns})$. In order to understand these trends, we analyze the $E_{p}$ dependence of the TOF spread by means of both analytical calculations as well as computer-based finite-element trajectory simulations using SIMION ${ }^{\circledR}$.

Owing to the complexity of the trajectories in the lens system, only the trajectory sections within the hemisphere are accessible to an analytical treatment. The impact of the lens system will be discussed further below. In general, the electron TOF spread within the hemisphere originates from (a) a finite energy resolution of the analyzer allowing electrons with slightly different kinetic energies to pass through the hemisphere and hit the detector at the same position, and (b) the simultaneous detection of electrons with the same kinetic energy traversing the analyzer on slightly different trajectories and arriving at the same detector position after traveling different path lengths. Both contributions are affected by a number of factors such as the widths of the hemisphere entrance and exit slits, the angular spread of electron velocity vectors upon entering the hemisphere, and the hemisphere pass energy. We use the analytical expressions given by Gießel et al. ${ }^{9}$ and Kugeler et $a l^{22}$ to estimate the impact of the analyzer energy resolution and electron trajectories, respectively. For pass energies $E_{p} \geq 50 \mathrm{eV}$, a hemisphere radius of $R_{0}=150 \mathrm{~mm}$, a (virtual) hemisphere exit slit width of $d$ $\leq 1 \mathrm{~mm}$, and electron angular spreads of the order of $2 \alpha$ $\leq 5^{\circ}$ at the hemisphere entrance aperture (see below), the trajectory induced TOF spread within the hemisphere exceeds the energy resolution induced TOF uncertainty by at least one order of magnitude. Consequently, we concentrate our analysis on the trajectory related TOF spread.

The dispersive plane angular spread $\pm \alpha$ of electrons entering the hemisphere and the spread of TOFs after which they arrive at the detector are linked by Kepler equations that describe the electron trajectories in a central potential. ${ }^{22}$ Note that we define the hemisphere entrance angle $\alpha$ consistent with previous works, ${ }^{9,22}$ i.e., it indicates the angular deviation of an electron trajectory from a tangential to the hemisphere at the entrance slit. Correspondingly, the total angular spread for an ensemble of trajectories with entrance angles $\alpha$ but different azimuthal angles around the lens system symmetry axis may be designated as $\pm \alpha$ or $2 \alpha$. Using the Kepler relationship between $\alpha$ and the TOF spread, the angular spreads of electrons entering the hemisphere are fit to the experimentally derived TOF spreads in Fig. 4(a) and displayed as blue squares in Fig. 4(b). Clearly, the width $2 \alpha$ of the hemisphere entrance angle distribution varies significantly with the retarding ratio. To further illustrate this effect, two black dashed lines in Fig. 4(a) mark the expected pass energy dependent TOF spreads for two constant angular spreads of $\alpha$ $= \pm 0.41^{\circ}$ and $\alpha= \pm 1.72^{\circ}$. The measured TOF spreads indicate that the experimental angular spreads vary between these two extremes.

Computer based trajectory simulations confirm the analytically derived trends. The solid blue circles in Fig. 4(b) are simulated angular spreads. They display a very similar overall rise with decreasing pass energy as the experimentally derived angles and vary between $2 \alpha=1.8^{\circ}$ (FWHM) and $2 \alpha=4.6^{\circ}$ (FWHM) at pass energies of $400 \mathrm{eV}$ and $50 \mathrm{eV}$, respectively. The simulated hemisphere TOF spreads are displayed as solid red circles in Fig. 4(a). Additionally, the total TOF spreads across the entire electron flight paths through the lens system and the hemisphere are shown as open red circles. The relatively small differences between the solid and open red circles indicate that the trajectories within the lens system contribute, in general, to a much smaller degree to the overall time resolution than the trajectories within the hemisphere. However, an important finding of this study is that the lens system indirectly contributes significantly to the overall time resolution of the setup by inducing a strong dependence of the hemisphere entrance angles on the retarding ratio. Figs. 4(a) and 4(b) demonstrate that this trend is reproduced by both the analytical interpretation of the experimental results and the trajectory simulations.

The root cause for the observed behavior can be understood by applying Liouville's theorem of constant emittance to the beam of electron trajectories within the lens system. ${ }^{24}$ It is known that for an electron retarding ratio $r=K E / E_{p}$ at the transition point between two regions along the flight path, the angular magnification $M_{a}$ and the lateral magnification $M_{l}$ 
of the trajectory bundle are connected by ${ }^{24}$

$$
M_{a} \cdot M_{l}=\sqrt{r} .
$$

The high transmission lens mode employed in this study is expected to lead to a constant lateral magnification $M_{l}$ of the electron trajectories. Therefore, the angular magnification $M_{a}$ should scale with the square root of the retarding ratio. The green diamonds in Fig. 4(b) were derived by applying this scaling law using the simulated angular spread at $E_{p}$ $=50 \mathrm{eV}$ as a reference. The simple estimate describes the simulated angular spreads very well and follows a similar trend as the experimental values. We note that the total TOF varies between $\sim 200 \mathrm{~ns}$ and $\sim 290 \mathrm{~ns}$ for pass energies of $400 \mathrm{eV}$ and $50 \mathrm{eV}$, respectively. Given the complexity of the lens system, the agreement between the simulated and the experimentally derived values for the TOF and angular spreads displayed in Fig. 4 is quite remarkable. The most notable discrepancies appear in the TOF spreads for pass energies $E_{p}$ $=200 \mathrm{eV}$ and $E_{p}=300 \mathrm{eV}$, for which the experimental values are less than half of the simulated spreads. We currently do not have a conclusive explanation for the local minimum in the TOF spread near these pass energies. One possibility is that the interaction volume was not perfectly aligned with the center axis of the spectrometer, which could limit the acceptance angle of the spectrometer and therefore reduce the temporal spread by reducing the average hemisphere entrance angle. The same mechanism could explain why all experimental TOF and hemisphere entrance angle spread values are slightly below the simulated ones. We note that the overall high temporal resolution of the setup enables (sub-)nanosecond tr-XPS measurements even for CW X-ray light sources, which may be interesting for laboratory-based XPS experiments.

\section{TRANSIENT SURFACE PHOTOVOLTAGE EFFECT IN Si(100)}

To demonstrate the capabilities of the tr-XPS setup, we study the dependence of the transient surface photovoltage $\left(\mathrm{SPV}^{33,34}\right)$ in a semiconductor on the pump laser fluence. The sample is a $13 \times 13 \mathrm{~mm}^{2}$ p-type (boron doped) $\mathrm{Si}(100)$ wafer covered by its native oxide layer. No additional surface preparation was performed prior to the tr-XPS measurements. Since the thickness of the native oxide layer (typically less than $1 \mathrm{~nm}^{35}$ ) is smaller than the electron inelastic mean free path, photoelectron signals from both the oxide layer and pure silicon can be detected in the XPS spectra. Time-resolved Si $2 p$ photoemission spectra of both bulk $\left(\mathrm{Si}^{0}\right)$ and oxide $\left(\mathrm{SiO}_{\mathrm{x}}\right)$ silicon were recorded. The kinetic energies of the collected electrons were between $324 \mathrm{eV}$ and $332 \mathrm{eV}$, the analyzer pass energy was $150 \mathrm{eV}$. The pump laser system (COHERENT Evolution 70) provided $240 \mathrm{~ns}$ (FWHM) long light pulses with a wavelength of $532 \mathrm{~nm}$ at a pulse repetition rate of $10 \mathrm{kHz}$. The laser spot size was $560 \times 420 \mu \mathrm{m}^{2}$ (FWHM). The X-ray spot size was $<100 \mu \mathrm{m}$ (FWHM) in both dimensions. The laser was not synchronized to the ALS bunch train; the synchrotron effectively operated as a CW X-ray source. We note that, while we have not made use of the ambient pressure capabilities of the setup in the time-domain experiments pre-
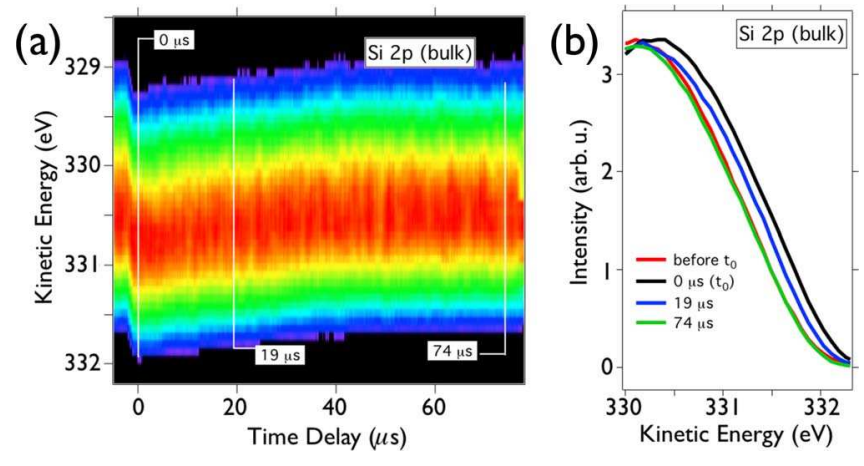

FIG. 5. (a) Time-resolved visible pump/X-ray probe XPS spectra of a $p$ doped $\mathrm{Si}(100)$ wafer in the range of the $\mathrm{S} 2 p$ photoline from bulk $\mathrm{Si}$ atoms. (b) Individual spectra extracted from (a) at different pump-probe time delays $(\mathrm{h} v=435 \mathrm{eV})$.

sented herein, the system is routinely used for ambient pressure experiments in the energy domain. ${ }^{23,27,28}$

Fig. 5(a) shows a false color representation of the $\mathrm{Si} 2 p$ trXPS map (for clarity only the bulk $\mathrm{Si}^{0}$ component is shown) obtained with a laser pump fluence of $12 \mu \mathrm{J} / \mathrm{cm}^{2}$. Fig. 5(b) compares several individual Si $2 p$ photoemission spectra extracted from Fig. 5(a) at different time delays as indicated by vertical white lines. The impact of the laser pulse on the photoemission peak position is clearly visible at time zero $(0 \mu \mathrm{s})$, where a $0.25 \mathrm{eV}$ shift of the Si $2 p$ peak toward higher kinetic energies occurs (Fig. 5(b), black curve). For longer time delays after the laser pulse, the peak position of the photoemission line gradually recovers (blue curve), reaching its initial value toward the end of the time delay range (green curve).

The origin of the SPV effect is linked to a static charge imbalance at the semiconductor surface. For a $p$-doped semiconductor, the electric field of the positive excess charge at the surface (emptied surface states) is screened by fixed, negatively charged acceptor states in the bulk. The resultant builtin electric field provokes a space charge region (SCR) with a characteristic downward bending of the electronic energy levels in the near-surface region ("band bending"). ${ }^{34}$ Since the width of the space charge layer (typically $\sim 100 \mathrm{~nm}$ ) significantly exceeds the escape depth for photoelectrons, tr-XPS exclusively probes electronic states within the SCR. Upon above-band-gap laser excitation, electron-hole pairs are generated and separated within the SCR, giving rise to a transient screening of the fixed charges. This transient screening translates into a corresponding time-dependent shift of the probed energy levels to lower binding energies ("band flattening"). ${ }^{33}$

Si $2 p$ tr-XPS maps similar to that displayed in Fig. 5 are recorded for a series of different pump laser fluences. The time-dependent photoelectron spectra are fit with two pseudo-Voigt peaks, representing the overlapping $\mathrm{Si} 2 p$ peaks of bulk $\mathrm{Si}^{0}$ and $\mathrm{SiO}_{\mathrm{x}}$ (ignoring the unresolved $\mathrm{Si} 2 p$ spinorbit splitting of $\sim 0.4 \mathrm{eV}$ ). The resulting temporal evolution of the $\mathrm{Si}^{0}$ peak position for a variety of pump laser fluences is shown in Fig. 6. The yellow curve indicates the unperturbed Si $2 p$ peak position without laser illumination. The impact of a laser pulse at time zero induces a time-dependent shift of the photoemission lines towards higher kinetic energies. The laser fluence impacts both the magnitude and the 


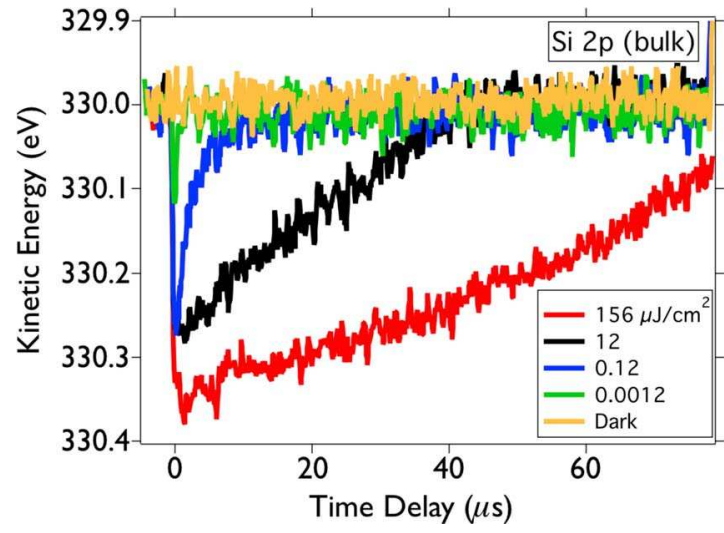

FIG. 6. Transient surface photovoltage dynamics of $\mathrm{SiO}_{\mathrm{x}} / \mathrm{Si}(100)$ as a function of pump laser fluence determined by the time-dependent $\mathrm{Si} 2 p$ (bulk) peak position $(\mathrm{h} v=435 \mathrm{eV})$.

temporal evolution of the SPV shift. The magnitude of the shift increases with increasing laser intensity, saturating at $\sim 350 \mathrm{meV}$. This saturation value is in good agreement with previous measurements. ${ }^{33}$

The time scale for the SPV decay after laser excitation is strongly affected by the laser fluence, exhibiting notably slower dynamics with increasing fluence: at a fluence of $0.12 \mu \mathrm{J} / \mathrm{cm}^{2}$, the SPV shift relaxes from $0.25 \mathrm{eV}$ to zero within the first $20 \mu$ s after excitation, whereas the SPV effect decays by only $\approx 50 \%$ within the same time window for $12 \mu \mathrm{J} / \mathrm{cm}^{2}$ laser fluence. A fluence-dependence of the SPV decay constant featuring the opposite trend, i.e., a faster SPV decay dynamics for higher laser fluences, has been observed by Widdra et al. in a similar experiment performed with $\mathrm{MHz}$ laser repetition rates. ${ }^{36}$ The SPV dynamics were explained within the framework of a thermionic emission model for laser-generated holes that tunnel through the timeand fluence-dependent potential barrier generated by the band bending at the surface. ${ }^{36,37}$ In this respect it is important to mention that - owing to the comparably low repetition rate - the transient SPV effect presented here completely relaxes between consecutive laser pulses even for the highest laser fluences in our experiment. This is evidenced by the constant kinetic energies at negative pump-probe delays in Fig. 6. In contrast, the $\mathrm{MHz}$ measurements by Widdra et al. ${ }^{36}$ were significantly affected by accumulated steady-state concentrations of laser-excited charge carriers in the SCR, which might influence the observed transient SPV response.

The temporal resolution of the laser-pump/X-ray-probe experiments presented here is defined by the $\sim 240$ ns duration (FWHM) of the laser pulses. We note, however, that the high temporal resolution of the setup, which can readily distinguish signals from different ALS light pulses in multi-bunch operating mode (Fig. 3(a)), also enables tr-XPS measurements with bunch-length limited temporal resolution when operated with a laser system that is synchronized with the ALS bunch train and that provides light pulses with durations much shorter than the ALS bunch length ( $\sim 70 \mathrm{ps}){ }^{38}$ The capability to perform time-resolved XPS experiments with pulse-length limited temporal resolution using trains of nanosecond to microsecond spaced X-ray pulses will be particularly useful for studies at future high repetition rate X-ray free electron laser (XFEL) light sources such as LCLS II and the European XFEL. As demonstrated here for pump-probe experiments using a $10 \mathrm{kHz}$ pump laser in combination with the $\sim 1.5-$ $500 \mathrm{MHz}$ bunch train of the ALS, time-tagging techniques will be able to take full advantage of the high X-ray repetition rates of next generation XFEL light sources without requiring similarly high pump laser repetition rates.

\section{SUMMARY}

A setup for sub-nanosecond time-resolved ambient pressure X-ray photoelectron spectroscopy based on the combination of a hemispherical electron kinetic energy analyzer and a fast time- and position-sensitive electron detector is presented. The intrinsic time resolution of the setup is determined by the TOF spread of electrons passing through the spectrometer with the same kinetic energy but on different trajectories. An average temporal resolution of $(780 \pm 20)$ ps is demonstrated for a pass energy $E_{p}=150 \mathrm{eV}$ and an electron kinetic energy range $K E=503-508 \mathrm{eV}$. This resolution is independent from the X-ray source and, therefore, enables trXPS studies with both pulsed and (laboratory-based) CW Xray light sources. While most of the TOF spread is acquired within the analyzer hemisphere, the electrostatic lens system that images the interaction volume onto the hemisphere entrance slit has decisive impact on the temporal resolution by defining the angular spread with which the electrons enter the hemisphere. In maximum transmission mode, the lens system induces a pronounced dependence of the temporal resolution on the retarding ratio through a significant variation of the hemisphere entrance angle spread. The scaling of the angular spread with the retarding ratio can be well approximated by Liouville's theorem of constant emittance along the electron beam path. The temporal resolution of the setup can be predicted by computer based trajectory simulations with a precision of $\leq 2 \mathrm{~ns}$. The time-tagging principle of the setup enables efficient use of the available X-ray fluence since all $\mathrm{X}$-ray photons independent of their intrinsic timing are used simultaneously in the measurement. The combination of highresolution XPS analyzers with fast time-tagging techniques as demonstrated herein may prove useful for experiments at future high-repetition rate XFEL light sources such as LCLS-II and the European XFEL.

\section{ACKNOWLEDGMENTS}

This work was supported by the U.S. Department of Energy, Office of Basic Energy Sciences, Chemical Sciences, Geosciences and Biosciences Division, through Contract No. DE-AC02-05CH11231. G.C., J.R., and R.A.K were supported by the U.S. Department of Energy, Office of Basic Energy Sciences, Division of Materials Sciences and Engineering under the same contract. O.G., S.N., and M.W.F. were supported by the Department of Energy Office of Science Early Career Research Program. The authors would like to thank Alan Fry and Wayne Polzin from SLAC National Accelerator Laboratory for their laser support. 
${ }^{1}$ L. Nugent-Glandorf, M. Scheer, D. A. Samuels, A. M. Mulhisen, E. R. Grant, X. Yang, V. M. Bierbaum, and S. R. Leone, Phys. Rev. Lett. 87, 193002 (2001)

${ }^{2}$ P. Siffalovic, M. Drescher, and U. Heinzmann, Europhys. Lett. 60, 924 (2002).

${ }^{3}$ T. Popmintchev, M.-C. Chen, D. Popmintchev, P. Arpin, S. Brown, S. Ališauskas, G. Andriukaitis, T. Balčiunas, O. D. Mücke, A. Pugzlys, A. Baltuška, B. Shim, S. E. Schrauth, A. Gaeta, C. Hernández-García, L. Plaja, A. Becker, A. Jaron-Becker, M. M. Murnane, and H. C. Kapteyn, Science 336, 1287 (2012).

${ }^{4}$ J. Chen and P. M. Rentzepis, J. Phys. Chem. Lett. 5, 225 (2014).

${ }^{5}$ W. Ackermann, G. Asova, V. Ayvazyan, A. Azima, N. Baboi, J. Bähr, V. Balandin, B. Beutner, A. Brandt, A. Bolzmann, R. Brinkmann, O. I. Brovko, M. Castellano, P. Castro, L. Catani, E. Chiadroni, S. Choroba, A. Cianchi, J. T. Costello, D. Cubaynes, J. Dardis, W. Decking, H. DelsimHashemi, A. Delserieys, G. D. Pirro, M. Dohlus, S. Düsterer, A. Eckhardt, H. T. Edwards, B. Faatz, J. Feldhaus, K. Flöttmann, J. Frisch, L. Fröhlich, T. Garvey, U. Gensch, C. Gerth, M. Görler, N. Golubeva, H. J. Grabosch, M. Grecki, O. Grimm, K. Hacker, U. Hahn, J. H. Han, K. Honkavaara, T. Hott, M. Hüning, Y. Ivanisenko, E. Jaeschke, W. Jalmuzna, T. Jezynski, R. Kammering, V. Katalev, K. Kavanagh, E. T. Kennedy, S. Khodyachykh, K. Klose, V. Kocharyan, M. Körfer, M. Kollewe, W. Koprek, S. Korepanov, D. Kostin, M. Krassilnikov, G. Kube, M. Kuhlmann, C. L. S. Lewis, L. Lilje, T. Limberg, D. Lipka, F. Löhl, H. Luna, M. Luong, M. Martins, M. Meyer, P. Michelato, V. Miltchev, W. D. Möller, L. Monaco, W. F. O. Müller, O. Napieralski, O. Napoly, P. Nicolosi, D. Nölle, T. Nuñez, A. Oppelt, C. Pagani, R. Paparella, N. Pchalek, J. Pedregosa-Gutierrez, B. Petersen, B. Petrosyan, G. Petrosyan, L. Petrosyan, J. Pflüger, E. Plönjes, L. Poletto, K. Pozniak, E. Prat, D. Proch, P. Pucyk, P. Radcliffe, H. Redlin, K. Rehlich, M. Richter, M. Roehrs, J. Roensch, R. Romaniuk, M. Ross, J. Rossbach, V. Rybnikov, M. Sachwitz, E. L. Saldin, W. Sandner, H. Schlarb, B. Schmidt, M. Schmitz, P. Schmüser, J. R. Schneider, E. A. Schneidmiller, S. Schnepp, S. Schreiber, M. Seidel, D. Sertore, A. V. Shabunov, C. Simon, S. Simrock, E. Sombrowski, A. A. Sorokin, P. Spanknebel, R. Spesyvtsev, L. Staykov, B. Steffen, F. Stephan, F. Stulle, H. Thom, K. Tiedtke, M. Tischer, S. Toleikis, R. Treusch, D. Trines, I. Tsakov, E. Vogel, T. Weiland, H. Weise, M. Wellhöfer, M. Wendt, I. Will, A. Winter, K. Wittenburg, W. Wurth, P. Yeates, M. V. Yurkov, I. Zagorodnov, and K. Zapfe, Nat. Photonics 1, 336 (2007)

${ }^{6}$ P. Emma, R. A. Akre, J. Arthur, R. Bionta, C. Bostedt, J. Bozek, A. Brachmann, P. Bucksbaum, R. Coffee, F. J. Decker, Y. Ding, D. Dowell, S. Edstrom, A. Fisher, J. Frisch, S. Gilevich, J. Hastings, G. Hays, P. Hering, Z. Huang, R. Iverson, H. Loos, M. Messerschmidt, A. Miahnahri, S. Moeller, H. D. Nuhn, G. Pile, D. Ratner, J. Rzepiela, D. Schultz, T. Smith, P. Stefan, H. Tompkins, J. Turner, J. Welch, W. White, J. Wu, G. Yocky, and J. Galayda, Nat. Photonics 4, 641 (2010).

${ }^{7}$ T. Ishikawa, H. Aoyagi, T. Asaka, Y. Asano, N. Azumi, T. Bizen, H. Ego, K. Fukami, T. Fukui, Y. Furukawa, S. Goto, H. Hanaki, T. Hara, T. Hasegawa, T. Hatsui, A. Higashiya, T. Hirono, N. Hosoda, M. Ishii, T. Inagaki, Y. Inubushi, T. Itoga, Y. Joti, M. Kago, T. Kameshima, H. Kimura, Y. Kirihara, A. Kiyomichi, T. Kobayashi, C. Kondo, T. Kudo, H. Maesaka, X. M. Maréchal, T. Masuda, S. Matsubara, T. Matsumoto, T. Matsushita, S. Matsui, M. Nagasono, N. Nariyama, H. Ohashi, T. Ohata, T. Ohshima, S. Ono, Y. Otake, C. Saji, T. Sakurai, T. Sato, K. Sawada, T. Seike, K. Shirasawa, T. Sugimoto, S. Suzuki, S. Takahashi, H. Takebe, K. Takeshita, K. Tamasaku, H. Tanaka, R. Tanaka, T. Tanaka, T. Togashi, K. Togawa, A. Tokuhisa, H. Tomizawa, K. Tono, S. Wu, M. Yabashi, M. Yamaga, A. Yamashita, K. Yanagida, C. Zhang, T. Shintake, H. Kitamura, and N. Kumagai, Nat. Photonics 6, 540 (2012).

${ }^{8}$ R. W. Schoenlein, S. Chattopadhyay, H. H. W. Chong, T. E. Glover, P. A Heimann, C. V. Shank, A. A. Zholents, and M. S. Zolotorev, Science 287, $2237(2000)$

${ }^{9}$ T. Gießel, D. Broöcker, P. Schmidt, and W. Widdra, Rev. Sci. Instrum. 74, 4620 (2003).

${ }^{10}$ A. M. March, A. Stickrath, G. Doumy, E. P. Kanter, B. Krässig, S. H. Southworth, K. Attenkofer, C. A. Kurtz, L. X. Chen, and L. Young, Rev. Sci. Instrum. 82, 073110 (2011).

${ }^{11}$ M. Chergui, Acta Crystallogr. A: Found. Crystallogr. 66, 229 (2010).

${ }^{12}$ C. Bressler and M. Chergui, Chem. Rev. 104, 1781 (2004).

${ }^{13}$ T. E. Glover, G. D. Ackermann, A. Belkacem, B. Feinberg, P. A. Heimann, Z. Hussain, H. A. Padmore, C. Ray, R. W. Schoenlein, and W. F. Steele, Nucl. Instrum. Methods Phys. Res. A 467-468, 1438 (2001).

${ }^{14}$ L. X. Chen, Annu. Rev. Phys. Chem. 56, 221 (2005).
${ }^{15}$ N. Huse, T. K. Kim, L. Jamula, J. K. McCusker, F. M. F. de Groot, and R. W. Schoenlein, J. Am. Chem. Soc. 132, 6809 (2010).

${ }^{16}$ B. E. Van Kuiken, N. Huse, H. Cho, M. L. Strader, M. S. Lynch, R. W. Schoenlein, and M. Khalil, J. Phys. Chem. Lett. 3, 1695 (2012).

${ }^{17}$ N. Pontius, C. Stamm, T. Kachel, R. Mitzner, T. Quast, K. Holldack, S. Khan, H. A. Dürr, and W. Eberhardt, in Ultrafast Phenomena XVI: Proceedings of the 16th International Conference, Palazzo dei Congressi Stresa, Italy, 9-13 June 2008, Springer Series in Chemical Physics Vol. 92, edited by P. Corkum, S. Silvestri, K. A. Nelson, E. Riedle, and R. W. Schoenlein (Springer, Berlin, 2009).

${ }^{18}$ N. Bergeard, M. G. Silly, D. Krizmancic, C. Chauvet, M. Guzzo, J. P. Ricaud, M. Izquierdo, L. Stebel, P. Pittana, R. Sergo, G. Cautero, G. Dufour, F. Rochet, and F. Sirotti, J. Synchrotron Radiat. 18, 245 (2011).

${ }^{19}$ A. S. Tremsin, O. H. W. Siegmund, J. S. Hull, J. V. Vallerga, J. B. McPhate, J. Soderstrom, J. W. Chiou, J. Guo, and Z. Hussain, IEEE Trans. Nucl. Sci. 54, 706 (2007).

${ }^{20}$ O. Höfert, C. Gleichweit, H. P. Steinrück, and C. Papp, Rev. Sci. Instrum. 84, 093103 (2013).

${ }^{21}$ D. M. Fritz, D. A. Reis, B. Adams, R. A. Akre, J. Arthur, C. Blome, P. H. Bucksbaum, A. L. Cavalieri, S. Engemann, S. Fahy, R. W. Falcone, P. H. Fuoss, K. J. Gaffney, M. J. George, J. Hajdu, M. P. Hertlein, P. B. Hillyard, M. Horn-von Hoegen, M. Kammler, J. Kaspar, R. Kienberger, P. Krejcik, S. H. Lee, A. M. Lindenberg, B. McFarland, D. Meyer, T. Montagne, E. D. Murray, A. J. Nelson, M. Nicoul, R. Pahl, J. Rudati, H. Schlarb, D. P. Siddons, K. Sokolowski-Tinten, T. Tschentscher, D. von der Linde, and J. B. Hastings, Science 315, 633 (2007).

${ }^{22}$ O. Kugeler, S. Marburger, and U. Hergenhahn, Rev. Sci. Instrum. 74, 3955 (2003).

${ }^{23}$ D. E. Starr, Z. Liu, M. Hävecker, A. Knop-Gericke, and H. Bluhm, Chem. Soc. Rev. 42, 5833 (2013).

${ }^{24}$ B. Wannberg, Nucl. Instrum. Methods Phys. Res. A 601, 182 (2009).

${ }^{25} \mathrm{H}$. Bluhm, K. Andersson, T. Araki, K. Benzerara, G. E. Brown, J. J. Dynes, S. Ghosal, M. K. Gilles, H. C. Hansen, J. C. Hemminger, A. P. Hitchcock, G. Ketteler, A. L. D. Kilcoyne, E. Kneedler, J. R. Lawrence, G. G. Leppard, J. Majzlam, B. S. Mun, S. C. B. Myneni, A. Nilsson, H. Ogasawara, D. F. Ogletree, K. Pecher, M. Salmeron, D. K. Shuh, B. Tonner, T. Tyliszczak, T. Warwick, and T. H. Yoon, J. Electron. Spectrosc. Relat. Phenom. 150, 86 (2006).

${ }^{26}$ A. Oelsner, M. Rohmer, C. Schneider, D. Bayer, G. Schönhense, and M. Aeschlimann, J. Electron. Spectrosc. Relat. Phenom. 178-179, 317 (2010).

${ }^{27}$ A. H. McDaniel, W. C. Chueh, A. Shavorskiy, T. Tyliszczak, H. Bluhm, K. F. McCarty, and F. E. Gabaly, ECS Trans. 58, 47 (2013).

${ }^{28}$ L. L. Patera, C. Africh, R. S. Weatherup, R. Blume, S. Bhardwaj, C. Castellarin-Cudia, A. Knop-Gericke, R. Schloegl, G. Comelli, S. Hofmann, and C. Cepek, ACS Nano 7, 7901 (2013).

${ }^{29}$ M. Huth, C.-T. Chiang, A. Trützschler, F. O. Schumann, J. Kirschner, and W. Widdra, Appl. Phys. Lett. 104, 061602 (2014).

${ }^{30}$ H. Rensmo, K. Westermark, S. Södergren, O. Kohle, P. Persson, S. Lunell, and H. Siegbahn, J. Chem. Phys. 111, 2744 (1999).

${ }^{31}$ K. R. Siefermann, C. D. Pemmaraju, S. Neppl, A. Shavorskiy, A. A. Cordones, J. Vura-Weis, D. S. Slaughter, F. P. Sturm, F. Weise, H. Bluhm, M. L. Strader, H. Cho, M.-F. Lin, C. Bacellar, C. Khurmi, J. Guo, G. Coslovich, J. S. Robinson, R. A. Kaindl, R. W. Schoenlein, A. Belkacem, D. M. Neumark, S. R. Leone, D. Nordlund, H. Ogasawara, O. Krupin, J. J. Turner, W. F. Schlotter, M. R. Holmes, M. Messerschmidt, M. P. Minitti, S. Gul, J. Z. Zhang, N. Huse, D. Prendergast, and O. Gessner, J. Phys. Chem. Lett. 5, 2753 (2014)

${ }^{32}$ C. Sun, G. Portmann, M. Hertlein, J. Kirz, and D. S. Robin, Phys. Rev. Lett. 109, 264801 (2012)

${ }^{33}$ J. P. Long, H. R. Sadeghi, J. C. Rife, and M. N. Kabler, Phys. Rev. Lett. 64, 1158 (1990).

${ }^{34}$ Z. Zhang and J. T. Yates, Chem. Rev. 112, 5520 (2012).

${ }^{35}$ M. Morita, T. Ohmi, E. Hasegawa, M. Kawakami, and M. Ohwada, J. Appl. Phys. 68, 1272 (1990)

${ }^{36}$ D. Bröcker, T. Gießel, and W. Widdra, Chem. Phys. 299, 247 (2004).

${ }^{37}$ W. Widdra, D. Bröcker, T. Gießel, I. V. Hertel, W. Krüger, A. Liero, F. Noack, V. Petrov, D. Pop, P. M. Schmidt, R. Weber, I. Will, and B. Winter, Surf. Sci. 543, 87 (2003).

${ }^{38}$ S. Neppl, A. Shavorskiy, I. Zegkinoglou, M. W. Fraund, D. Slaughter, T. Troy, M. P. Ziemkiewicz, M. Ahmed, S. Gul, B. Rude, J. Z. Zhang, A. S. Tremsin, P.-A. Glans, Y.-S. Liu, C. H. Wu, J. Guo, M. Salmeron, H. Bluhm, and O. Gessner, Faraday Discuss. (published online). 